\title{
Franca Brambilla Ageno e l'Accademia della Crusca
}

Elisabetta Benucci

PUBBLICATO: 31 DICEMBRE 2019

E un lungo rapporto quello che ha legato, e lega ancora oggi, Franca Brambilla Ageno (r9izI995), raffinata filologa e linguista, all'Accademia della Crusca [I]. Un lungo rapporto che è iniziato nel lontano 1939 e che idealmente dura ancora oggi, grazie alle sue carte riunite in un corposissimo fondo conservato nell'Archivio accademico ${ }^{\mathrm{I}}$ [2]. Il fondo, che è stato donato con grande liberalità nel 1999 dalla figlia Elena Brambilla, è già stato ordinato e inventariato da Caterina Canneti nel 2016 ed è disponibile in rete nella banca dati dell'Accademia Archivio Digitale ${ }^{2}$ I3l. $_{\text {I }}$

Grazie a queste carte, è possibile entrare nel laboratorio filologico e linguistico di Franca Ageno e poterne ricostruire minuziosamente le attività, le ricerche, le consultazioni di opere. Un lavoro, quello di Franca Ageno, che non finiva mai, perché c'era sempre qualcosa da investigare, da approfondire, da aggiungere. Si possono cosi ripercorrere la genesi e la preparazione dei suoi più importanti scritti: dalle edizioni commentate di autori antichi (Iacopone, Pulci, Sacchetti, Panuccio del Bagno)3 [4], al fortunato manuale di filologia (L'edizione critica dei testi volgari) ${ }^{4}$, alle indagini sulla sintassi antica (Il verbo nellitaliano antico) 5 . Né va dimenticato che per più di un ventennio Franca Ageno fu impegnata nell'edizione del Convivio dantesco, pubblicato postumo nel $1995^{6}[5]$.

Dobbiamo preliminarmente ricordare che già hanno visto la luce importanti studi sull'attività di Franca Ageno: dal numero monografico a lei dedicato di "Schede umanistiche" del i997 (con testimonianze di Ezio Raimondi, Guglielmo Gorni, Carlo Delcorno, Domenico De Robertis, Paolo Bongrani), al contributo di Nicoletta Leone del 2006, al convegno Tra filologia e storia della lingua italiana. Per Franca Brambilla Ageno, a cura di Andrea Canova del 2015, ai vari studi in rivista di Paolo Pellegrini del 2016 (intorno alla progettata e mai realizzata edizione delle opere del Sacchetti), di Alice Ferrari del 2016 (sulla vicenda editoriale dell'edizione critica del Morgante del Pulci), oltre i suggestivi ricordi di Maria Corti, affidati alle pagine del volume Dialogo in pubblico del $1995^{7}$.

Tuttavia, a quel che ci risulta, non è stata ancora ripercorsa la storia delle relazioni, che vedremo molto importanti, fra Franca Ageno e la Crusca. Rapporto che ben si ricostruisce, oltre che sulle sue carte, anche con i documenti che si trovano nell'Archivio storico dell'Accademia e che sono stati inventariati da Giulia Marucelli ${ }^{8}$.

Qualche dato, allora, che poi approfondiremo: comandata giovanissima alla Crusca nel i939, fu collaboratrice assidua degli "Studi di Filologia Italiana" (unica studiosa a pubblicarvi ben diciotto articoli fra il I950 e il I962), poi consulente dell'Ufficio filologico dell'Opera del Vocabolario fra il I967 e il I969, infine prima donna a essere ammessa nel Novecento come socia corrispondente nel 1970, poi accademica ordinaria nel ig9o.

È dunque l'eccezionalità di questa personalità femminile nei suoi rapporti con la Crusca che qui vogliamo far emergere, poiché Franca Ageno risulta essere la prima donna a collaborare così strettamente con l'Accademia. È vero, nella seconda metà dell'Ottocento due donne erano state elette accademiche corrispondenti, Caterina Franceschi Ferrucci ed Ersilia Caetani Lovatelli: ma era stata solo un'elezione che aveva dato merito alla Crusca, per aver accolto finalmente donne in un consesso 
da sempre formato di soli accademici uomini, e lustro alle due signore. Di fatto non c'era stata alcuna relazione di lavoro fra queste studiose e la Crusca?.

Aveva ventisei anni Franca Ageno quando partecipò nel 1939 al concorso per un comando all'Accademia della Crusca, presso il Centro di studi di filologia italiana.

Originaria di Reggio Emilia, dove era nata il 27 maggio I9ı3, Franca Ageno si era laureata all'Università di Genova, sotto l'insegnamento di Achille Pellizzari e di Alfredo Schiaffini ${ }^{\mathrm{IO}}$. Insegnava già materie letterarie al R. Ginnasio "Colombo" di Genova e decise di tentare il concorso in Crusca. Il risultato, considerati i tempi, fu sorprendente e la studiosa risultò di gran lunga prima, come testimonia il verbale accademico dell'adunanza del 25 novembre $1939^{\text {II }}$ :

...dei quattro concorrenti il più meritevole è senza confronto la dottoressa Ageno Franca, le cui pubblicazioni, essendo di carattere critico a costituzione di testi, mostrano conoscenza della lingua antica e metodo sicuro; segue il dott. Caretti Lanfranco, che ha buoni titoli [...]. Quindi l'Accademia designa per due posti (in attesa della nomina ministeriale) i proff. Ageno e Caretti.

All'epoca, Franca Ageno aveva pubblicato l'edizione Il Bianco da Siena. Notizie e testi inediti, frutto della sua tesi di laurea ${ }^{\mathrm{I} 2}$; la tesi sul Bianco era uno studio critico condotto con un parallelo tra il modestissimo Bianco da Siena e Iacopone da Todi, "il più grande poeta, dopo Dante, e delle nostre origini"'I3.

Il Centro di studi di filologia italiana era, nel I939, un'istituzione recente. Era stato infatti creato nell'estate del 1937, allo scopo "di promuovere lo studio e la edizione critica degli antichi testi e degli scrittori classici della letteratura italiana dalle origini al secolo XX"I4. A dirigere il Centro era stato chiamato, nei primi mesi del 1938, Luigi Foscolo Benedetto.

In questo rinnovato fervore di studi filologici e linguistici (seguito allinterruzione dei lavori lessicografici del 1923), già nel settembre 1937 erano stati designati due comandati, Vittore Branca e Gianfranco Contini. Erano seguiti i comandi di Franca Ageno e di Lanfranco Caretti, che furono a disposizione del Centro dal 16 gennaio i940 fino al I5 ottobre 1942.

Franca Ageno varcò la soglia di Palazzo Pucci, in via de' Pucci al numero 4, ai primi di gennaio del I940. Da circa un anno era questa la sede provvisoria dell'Accademia.

All'appena comandata Ageno, gli Accademici proposero di occuparsi di qualche testo molto antico, come stava facendo Vittore Branca, impegnato sul testo dell'Amorosa visione di Boccaccio. La studiosa subito si era dichiarata disponibile a tentare l'edizione di Iacopone da Todi come si legge nel verbale

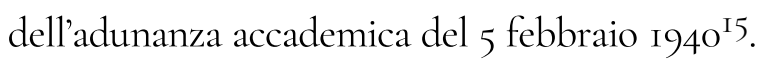

Trasferitasi a Firenze per il suo nuovo incarico, Franca Ageno aveva trovato un alloggio in un educandato di via Borgo Pinti, al numero ${ }_{5}$ [6], vicino alle più importanti Biblioteche della città, dove giornalmente si recava a studiare. Con quella passione e meticolosità che contraddistingueranno sempre i suoi studi di filologa e di linguista, affronto il lavoro che le era stato assegnato e che si concretizzerà, più di dieci anni dopo, nel 1953, nell'edizione Laudi, Trattato e Detti di Iacopone, per i tipi di Le Monnier, ma anticipata da diversi saggi preparatori pubblicati in riviste ${ }^{16}$.

A Firenze Franca Ageno ebbe occasione di conoscere i grandi rinnovatori del dantismo italiano e i fondatori della nuova filologia italiana - Barbi, Mazzoni, Busnelli, Vandelli, Maggini - e strinse saldi 
legami di amicizia con alcuni giovani colleghi, soprattutto con Contini e con Branca, il quale la ricorderà "coi grandi occhi azzurri fissamente intenti su codici", al lavoro "sui banchi delle più severe e ricche biblioteche e al Centro di filologia italiana dell'Accademia della Crusca"' 7 .

Intanto la filologa si era unita in matrimonio con Arturo Brambilla, insegnante, scrittore, amico caro di Dino Buzzati. Secondo le leggi di quel periodo, Franca Brambilla Ageno aveva anche ricevuto il "premio di nuzialità disposto dal Duce" per gli insegnanti, 4.ooo lire con "vaglia cambiario emesso dalla Banca di Italia", che l'Accademia le aveva consegnato ${ }^{\mathrm{I}}$.

I due sposi si erano stabiliti a Milano. Franca Brambilla Ageno si vedeva suo malgrado costretta a rinunciare all'esperienza fiorentina che tanto la gratificava per poter stare vicino al marito. Aveva anche cercato di trovare un compromesso, proponendo all'Accademia di poter continuare gli studi tanto amati, senza risiedere a Firenze. L'Accademia, pur riconoscendo i meriti della studiosa, non aveva accettato.

La decisione era stata presa ed era definitiva. Accanto al breve biglietto con il quale annunciava la sua rinuncia, Franca Brambilla Ageno sentì la necessità di scrivere una lettera, con la sua chiara ed elegante grafia, a Luigi Foscolo Benedetto, che tanto l'aveva sostenuta e lodata nel suo incarico ${ }^{19}$ :

[...] Mentre Le esprimo il mio rincrescimento, molto vivo, di aver dovuto rinunciare a far parte del Centro, presso cui lavoravo con vero entusiasmo, ringrazio ancora una volta Lei delle benevolenza con cui mi ha accolta e trattata, e degli aiuti e dei suggerimenti preziosi di cui mi è stata larga durante i mesi del mio soggiorno fiorentino, e La prego di scusarmi se, involontariamente, posso averLe procurato qualche fastidio.

Continuerò certamente a lavorare intorno all'argomento che mi era stato assegnato, e se, a guerra finita, potrò tornare qualche volta a Firenze, a consultare i "miei" manoscritti, spero che potrò venire a salutare Lei, che tanto ha fatto per me in questo periodo.

Non poteva, e non doveva, essere un "addio" alla Crusca e alla filologia dell'Accademia. E infatti alcuni anni dopo, Franca Brambilla Ageno diventerà per un certo periodo la più assidua collaboratrice degli "Studi di Filologia Italiana", la prestigiosa rivista che l'Accademia pubblicava fin dal 1927.

Ben diciotto, infatti, sono gli scritti che Franca Brambilla Ageno pubblico fra il I950-1962 negli "Studi di Filologia Italiana", a sottolineare l'affermazione non comune di questa studiosa in un periodo del Novecento, caratterizzato da un quasi deserto di presenze femminili nella filologia e nella linguistica. Li elenchiamo di seguito in ordine cronologico (con l'aggiunta dell'ultimo articolo edito da Franca Ageno nel 1984 che portano complessivamente i saggi a diciannove) $[7]$ :

I. Ancora per il testo delle "Laudi" di Iacopone da Todi, VIII, I950, pp. 5-28.

2. Le tre redazioni del "Morgante", IX, I95I, pp. 5-37.

3. Riboboli trecenteschi, X, I952, pp. 4I3-454.

4. Per una nuova edizione della "Battaglia" del Sacchetti, XI, I953, pp. 245-255.

5. Per una nuova edizione delle "Rime" del Sacchetti, XI, I953, pp. 257-320.

6. Metaplasmi nominali nell'antico toscano e umbro, XII, I954, pp. 313-323.

7. "Fare" e "usare" modali, XII, I954, pp. 325-333.

8. La lingua della Cronaca todina di Ioan Fabrizio degli Atti, XIII, I955, pp. I67-227.

9. L'uso pleonastico della negazione nei primi secoli, XIII, I955, pp. 339-36I.

Io. Ancora sui bestiari del "Morgante", XIV, I956, pp. 485-493.

II. Per una semantica del gergo, XV, I957, pp. 4OI-437. 
I2. Per il testo del "Trecentonovelle", XVI, I958, pp. 193-274.

I3. Un saggio di furbesco del Cinquecento, XVII, I959, pp. 22I-237.

I4. Le frasi proverbiali di una raccolta manoscritta di Lionardo Salviati, XVII, I959, pp. 239-274.

15. Due contributi sintattici, XVII, I959, pp. 295-318.

16. Ancora per la conoscenza del furbesco antico, XVIII, i960, pp. 79-Ioo.

17. Saggio di edizione critica di una laude trecentesca, XX, I962, pp. 3I-74.

18. Tre studi quattrocenteschi, XX, 1962, pp. 75-98.

19. Due note testuali sul "Discorso intorno alla nostra lingua" del Machiavelli, XLII, I984, pp. I6I-I64.

La maggior parte di questi saggi editi negli "Studi di Filologia Italiana" attenevano ai lavori in corso della filologa. Confermano anche che il campo di elezione dei suoi studi era il periodo letterario che andava dalle origini alla fine del Quattrocento e che quell'ambito veniva coltivato senza prevenzioni particolari; prosa e poesia - quest'ultima peraltro prevalente -, produzione laudistica e lirica d'arte, cantari in ottave e "riboboli trecenteschi", grandi autori e autori anonimi, lingua zerga e parlar furbesco, novelle ed egloghe volgari, compatti canzonieri o codici miscellanei dei più misti e avventurosi: niente pareva a Franca Ageno estraneo al suo gusto e al riparo dalla sua filologica inquisizione.

Il primo scritto pubblicato sugli "Studi di Filologia Italiana", Ancora per il testo delle "Laudi" di Iacopone da Todi, annunciava l'imminente uscita dell'edizione del i953. Data alle stampe dopo lunghissima attesa, l'edizione di Iacopone apparve in una collana per un pubblico non specialistico, non aveva apparato critico, mentre la classificazione dei manoscritti veniva concentrata in una nota di due pagine $^{20}$. Lopera, nonostante qualche riserva, le valse però l'apprezzamento di Gianfranco Contini, che la chiamò a lavorare in vista di quell'antologia che sarebbe diventata i Poeti del Duecento, in particolare per la sezione iacoponica e per quella delle Laude cortonesi ${ }^{21}$. Questa volta, per gli scritti di Iacopone, Franca Ageno poteva progettare una vera e propria edizione critica, una seconda edizione, alla quale iniziò subito a lavorare, fin dal i953. Lo testimoniano le tante carte, appunti, notazioni conservati tra le sue carte in Crusca [8]. Non è chiaro fino a quando questo progetto ebbe forza; la seconda edizione infatti non fu portata a termine, perché probabilmente soppiantata dal grande impegno per il Convivio ${ }^{22}[\mathbf{9}]$.

Anche per le altre edizioni che stava preparando, Franca Ageno pubblico anticipazioni sugli "Studi di Filologia Italiana": si pensi agli scritti su Le tre redazioni del "Morgante", del I951; oppure agli scritti Per una nuova edizione della "Battaglia" del Sacchetti, e Per una nuova edizione delle "Rime" del Sacchetti, entrambi del I953; si pensi infine all'articolo Per il testo del "Trecentonovelle", del i958. E non solo dava anticipazioni; ma tornava a puntualizzare sui testi già editi, con nuove investigazioni critiche. Così dopo l'edizione del Morgante data alle stampe nel I955 [Io], opera che aveva segnato quasi esattamente il mezzo del cammino della vita della curatrice, Franca Ageno affidava a una breve nota del I956, dal titolo Ancora sui bestiari del "Morgante", un'ultima proposta testuale ${ }^{23}$.

La filologia di Franca Ageno era fatta anche di infiniti scritti minori, che abbondarono su riviste importanti, a partire da "Lingua Nostra" fino ad arrivare agli "Studi Danteschi" e agli "Studi di Grammatica italiana". Sugli "Studi di Filologia Italiana", nello specifico, compaiono postille, annotazioni sintattiche (si pensi ai Riboboli trecenteschi, del 1952 e ai Due contributi sintattici, del 1959); compaiono annotazioni linguistiche (ci riferiamo ai saggi sulla Lingua della Cronaca todina di Ioan Fabrizio degli Atti, del 1955, o alle Frasi proverbiali di una raccolta manoscritta di Lionardo Salviati, del I959); compaiono nuove proposte e note, appunti e poscritti, contributi per l'edizione di questo o di quel testo, come nel caso del Saggio di edizione critica di una laude trecentesca, del $1962 .{ }^{24}$ 
L'assidua collaborazione terminò nel I962; solo nel 1984, lo abbiamo ricordato, Franca Ageno tornerà a pubblicare sugli "Studi di Filologia Italiana" un ultimo articolo: le Due note testuali sul "Discorso intorno alla nostra lingua" del Machiavelli. Nel 1977, tuttavia, i "Quaderni degli Studi di Filologia Italiana" avevano accolto la sua edizione delle Rime di Panuccio del Bagno. Nel frattempo erano arrivate dall'Accademia le richieste di collaborare, tra il I967 e il I969, al "Tesoro della lingua antica" e al rinato "Vocabolario della lingua italiana"25.

Franca Ageno fu eletta socia corrispondente della Crusca nella riunione del Collegio accademico del I 5 maggio I970, alla presenza di Giacomo Devoto, presidente, e di Carlo Alberto Mastrelli, segretario ${ }^{26}$. Si dovevano infatti eleggere due soci corrispondenti nazionali e Franca Ageno risulto avere avuto, insieme a Piero Fiorelli, le maggiori preferenze 27 . Due decenni dopo fu accolta fra gli accademici ordinari nella seduta del Consiglio direttivo del 6 luglio 1990, presidente Giovanni Nencioni, segretario Piero Fiorelli28.

Qualche anno dopo la sua scomparsa, avvenuta il I3 ottobre 1995, la figlia Elena Brambilla, docente di storia moderna all'Università di Milano, decise di donare, nel luglio del 1999 e su invito di Domenico De Robertis, una parte cospicua delle carte di Franca Ageno all'Archivio dell'Accademia della Crusca. Materiale che giunse in Accademia nell'ottobre successivo, come le comunicava il presidente di allora, Giovanni Nencioni.

Ho avuto l'ultimo contatto per posta elettronica con la prof.ssa Elena Brambilla nell'ottobre del 2016 per annunciarle che il fondo era stato opportunamente descritto, collocato in un armadio apposito e che era a disposizione degli studiosi.

Elena Brambilla mi rispose con una mail, dai toni quasi entusiastici, dove ai sentiti ringraziamenti univa questo auspicio: "spero, persino, offrendomi se occorresse di collaborare in tutto con voi, che si possa organizzare una giornata di studio per valorizzare quest'aggiunta al patrimonio archivistico dell'Accademia" [II].

Vogliamo allora immaginare che Elena Brambilla, scomparsa nel gennaio 20I8, avrebbe accolto con soddisfazione il ricordo della filologa Franca Ageno, affidato alla manifestazione organizzata dall'Accademia della Crusca.

Note:

I. Archivio dell'Accademia della Crusca, Firenze (di seguito indicato con la sigla ACF), Fondo Franca Brambilla Ageno, filze i385-I393bis, I4I7-I4I7ter.

2. All'indirizzo: www.adcrusca.it (nello specifico, "Fondo aggregato Franca Brambilla Ageno": http://www.adcrusca.it/theke/treeview2.asp?IDOggetto=3392 ). Cfr. inoltre Caterina Canneti, Il Fondo Brambilla Ageno all'Accademia della Crusca, in "Studi di Erudizione e di Filologia Italiana", VI, 20I7, pp. 283-337.

3. Ci riferiamo alle note edizioni: Iacopone da Todi, Laudi, Trattato e Detti, a cura di Franca Ageno, Firenze, Le Monnier, 1953; Luigi Pulci, Morgante, a cura di Franca Ageno, Milano-Napoli, R. Ricciardi, I955; Le rime di Panuccio del Bagno, a cura di Franca Brambilla Ageno, Firenze, presso l'Accademia della Crusca, I977 ("Quaderni degli Studi di Filologia Italiana"); Franco Sacchetti, Il libro delle rime, edited by Franca Brambilla Ageno, Firenze-Perth, L.S. Olschki - University of Western Australia Press, I99o. 
Preziosa, infine, è la Bibliografia di Franca Brambilla Ageno curata da Carlo Delcorno, edita in Franca Brambilla Ageno, Studi danteschi, con una premessa di Carlo Delcorno, Padova, Antenore, I990, pp. 263-285, che arriva fino al 1985 .

4. Franca Brambilla Ageno, L'edizione critica dei testi volgari, Padova, Antenore, I975 (2 $\underline{\underline{a}}$ ed. ampliata I984).

5. Eadem, Il verbo nell'italiano antico. Ricerche di sintassi, Milano-Napoli, Ricciardi, I964.

6. Dante Alighieri, Convivio, a cura di Franca Brambilla Ageno, Firenze, Le Lettere, 1995, 2 voll. (dei quali il primo di Introduzione occupa due tomi) ("Edizione Nazionale delle Opere di Dante Alighieri", a cura della Società Dantesca Italiana).

7. Rispettivamente: In memoria di Franca Brambilla Ageno. Testimonianze e studi per una Maestra, a cura dell'Università degli Studi di Parma, Istituto di Filologia moderna, Atti della giornata di studio, Parma, 24 ottobre 1996, in "Schede umanistiche", n.s., I, I997; Nicoletta Leone, Filologia tra "croci" e "delizie": Franca Ageno e Maria Corti, in "Nuova Antologia", 2237, 2006, pp. 199-207; Tra filologia e storia della lingua italiana. Per Franca Brambilla Ageno, a cura di Andrea Canova, Roma, Edizioni di Storia e Letteratura, 2015 (con una sezione dedicata alla descrizione della biblioteca di Franca Ageno, donata da Elena Brambilla insieme all'archivio epistolare della madre, alla Biblioteca dell'Università Cattolica del Sacro Cuore di Brescia); Paolo Pellegrini, Tra le carte di Franca Brambilla Ageno: ledizione delle "Opere" di Franco Sacchetti, in "Storie e Linguaggi", II, 2016, I, pp. I45-I58; Alice Ferrari, «La "passione" del Morgante». Franca Brambilla Ageno e l'edizione critica del poema pulciano, in "Studi di Erudizione e di Filologia Italiana", V, 20I6, pp. 345-37I; Maria Corti, Dialogo in pubblico. Intervista di Cristina Nesi, Milano, Rizzoli, 1995, pp. 59-62.

8. Fondo Documenti del Novecento (relazioni sulle attività dell'Accademia, atti amministrativi, verbali, lettere), all'indirizzo di Archivio Digitale: http://www.adcrusca.it/theke/treeview2.asp? IDOggetto $=10558$

9. A tal proposito cfr. Elisabetta Benucci, "Il piu bel fior ne coglie". Donne accademiche e socie della Crusca, in Italia linguistica: gli ultimi 150 anni. Nuovi soggetti, nuove voci, un nuovo immaginario, a cura di Elisabetta Benucci e Raffaella Setti, presentazione di Nicoletta Maraschio, Firenze, Accademia della Crusca - Le Lettere, 20II pp. 2I-26; di Franca Ageno si parla alle pp. 26-28.

Io. Per le notizie biografiche sulla studiosa ci atteniamo alle pagine di Carlo Delcorno, Necrologio. Franca Brambilla Ageno (Reggio Emilia 1913 - Milano 1995), in "Giornale storico della letteratura italiana", CLXXIII, I996, I, pp. 315-320.

II. ACF, filza 384, Verbali I7, p. 52 .

I2. Franca Ageno, Il Bianco da Siena. Notizie e testi inediti, Genova, Società editrice Dante Alighieri, I939.

I3. Ivi, p. XXVIII. Cfr. anche Carlo Delcorno, Il contributo di Franca Brambilla Ageno agli studi di letteratura religiosa medievale, in In memoria di Franca Brambilla Ageno. Testimonianze e studi per una Maestra, cit., pp. 33-47 (in particolare pp. 35-36).

I4. Si cita dal "Regolamento". La documentazione relativa all'istituzione del Centro di studi di filologia italiana è conservata in ACF, Fondo Novecento, filza 1567 , cartella 27. 
I5. ACF, filza 384, Verbali I7, p. 50.

I6. Si vedano, per esempio, Franca Ageno, Per il testo delle laudi di Iacopone da Todi, in "La Rassegna", LI-LVI, I943-I948, pp. 3-47; Eadem, Modi stilistici delle laudi di Iacopone, in "La Rassegna d'Italia", I, I946, pp. 20-29; Eadem, Per un commento a Iacopone da Todi, in "Convivium", n.s., I, I950, pp. 73-96. Ne darà anticipazione, come diremo più oltre, anche negli "Studi di Filologia Italiana".

I7. Vittore Branca, E ora gustiamo le pagine del «Convivio», "Il Sole-24 Ore", 24 dicembre 1995.

I8. Tutta la documentazione, compresa una breve lettera autografa dell'Ageno per il ricevimento del vaglia ("Milano, 2 settembre I940. Via Bianca di Savoia 15"), in ACF, Fondo Novecento, filza I567, cartella 28 , ai numeri d'ordine $26,39,40$.

19. Lettera autografa in ACF, Fondo Novecento, filza 1567 , cartella 28, numero d'ordine 36.

20. Carlo Delcorno, Il contributo di Franca Brambilla Ageno agli studi di letteratura religiosa medievale, cit., pp. 42-43.

2I. Di Franca Ageno infatti fu la cura di 15 laudi cortonesi, 25 laudi di Iacopone e dei Proverbi di Ser Garzo per i Poeti del Duecento, a cura di Gianfranco Contini, II, Milano-Napoli, Ricciardi, pp. I2-59; 67I66; 296-313. Sull'argomento cfr. Andrea Canova, Dal laboratorio di Franca Brambilla Ageno, cit., pp. 8789. I «Proverbi» di Ser Garzo furono riediti molti anni dopo negli "Studi Petrarcheschi”, n.s., I, I984, pp. I-37.

22. Andrea Canova, Dal laboratorio di Franca Brambilla Ageno, cit., p. 94.

23. Cfr. "Studi di Filologia Italiana" XIV, I956, pp. 485-493.

24. Per i riferimenti bibliografici di ogni saggio qui ricordato e stampato da Franca Ageno sugli "Studi di Filologia Italiana", vd. la lista elencata in precedenza.

25. Per la documentazione cfr. ACF, Fondo Franca Brambilla Ageno, filza iz88bis, cartella «Crusca. Corrispondenza per Vocabolario». L'episodio, con la pubblicazione di alcuni documenti, è ricostruito in Domenico De Robertis, L'edizione del "Morgante", in In memoria di Franca Brambilla Ageno. Testimonianze e studi per una Maestra, cit., pp. 62-64.

26. ACF, filza 387, Verbali 20, p. 157 .

27. La documentazione in ACF, Fondo Novecento, busta I2, fascicolo II, intestato Accademici ordinari. Franca Brambilla Ageno (si tratta di corrispondenza in arrivo e in partenza e comunicazioni relative al ruolo di accademico).

28. ACF, filza 394, Verbali 20, p. I8I. 


\section{Immagini:}

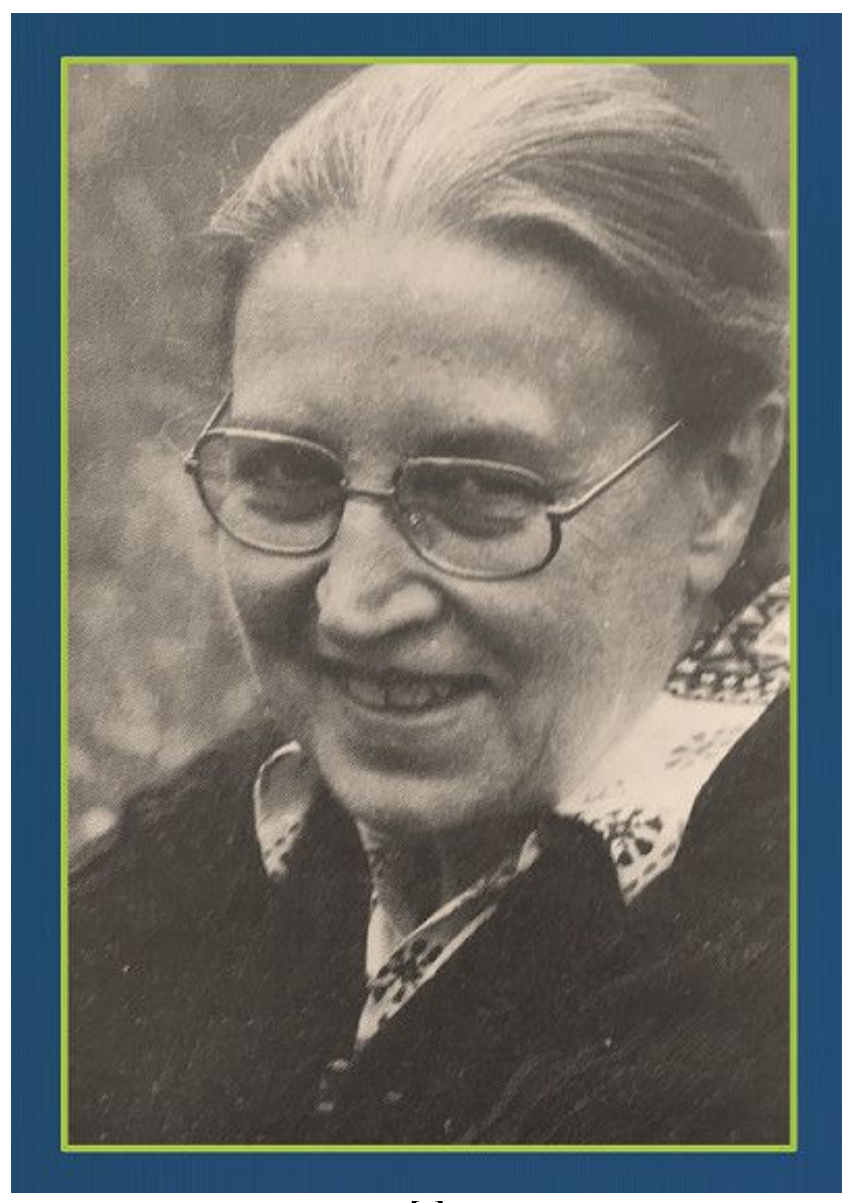

[I]

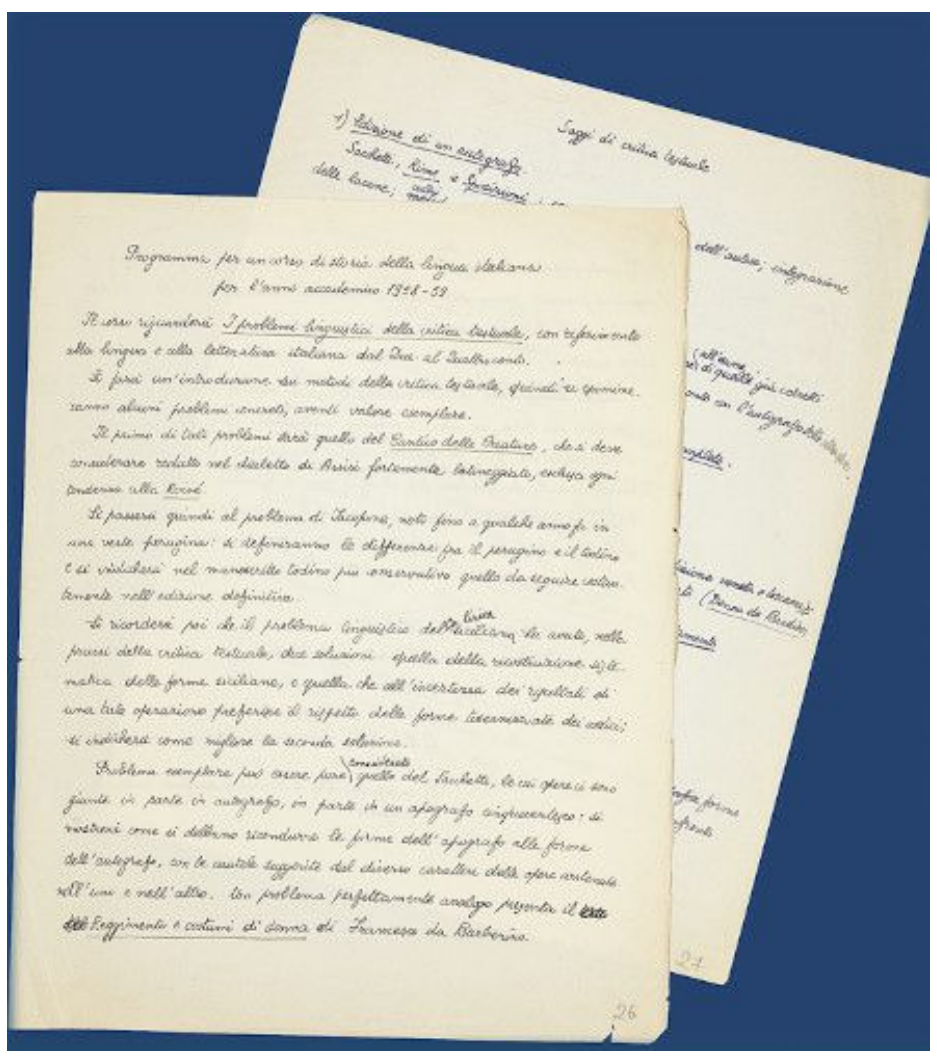




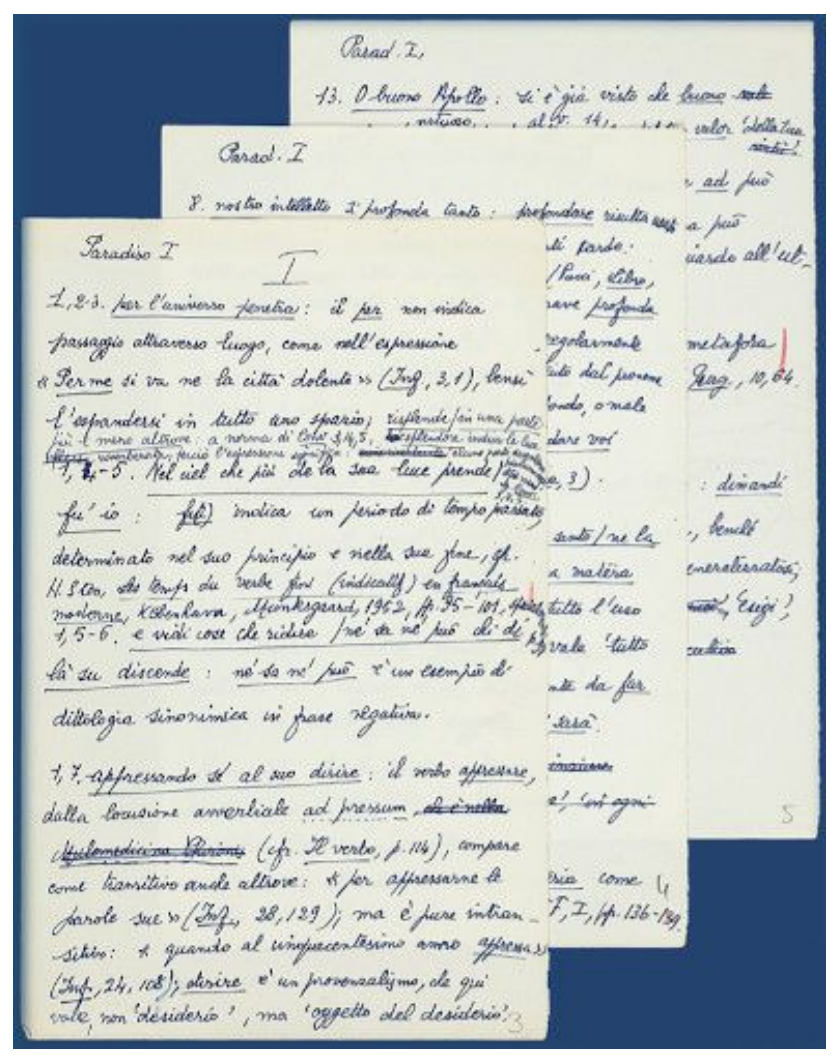

[2a]

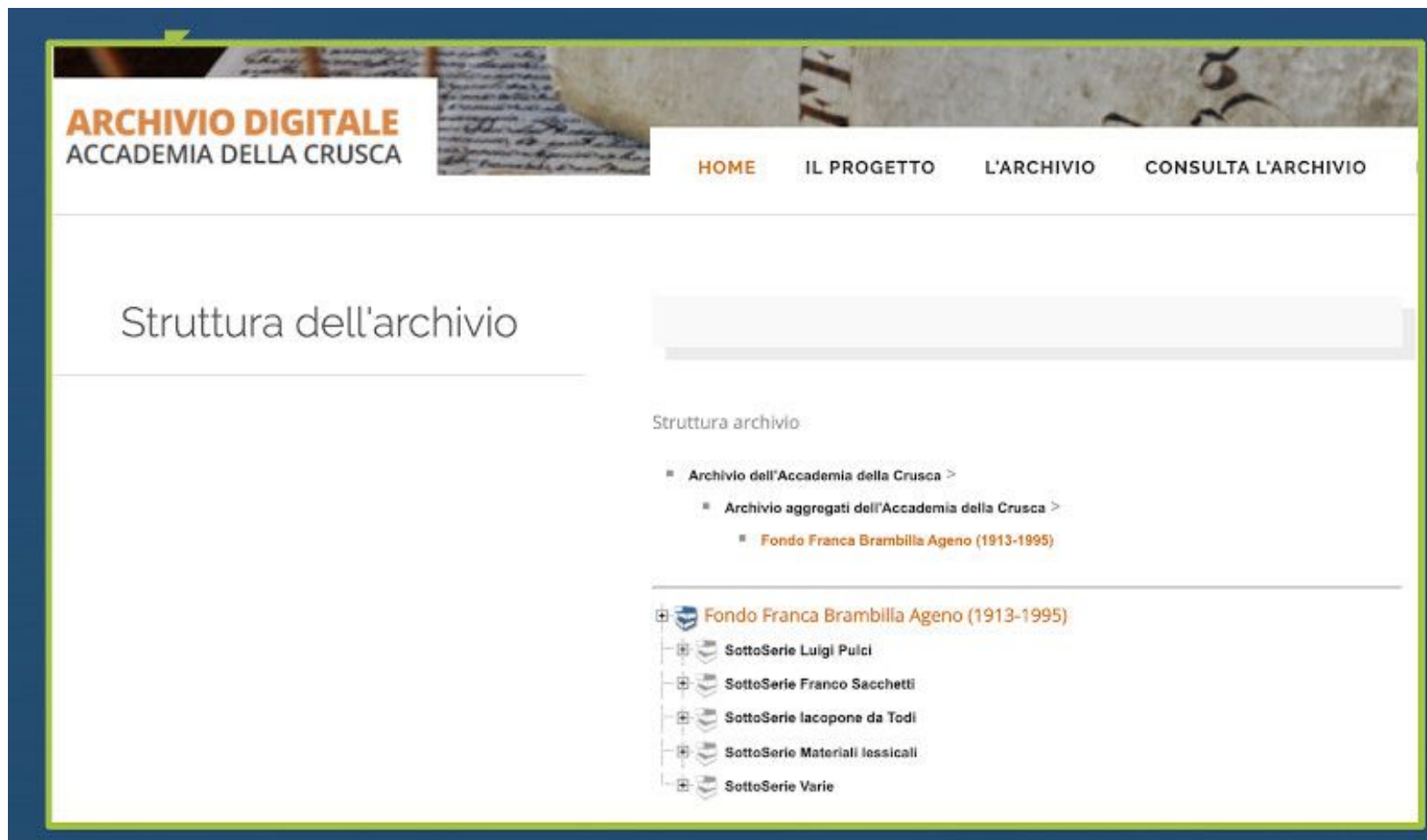




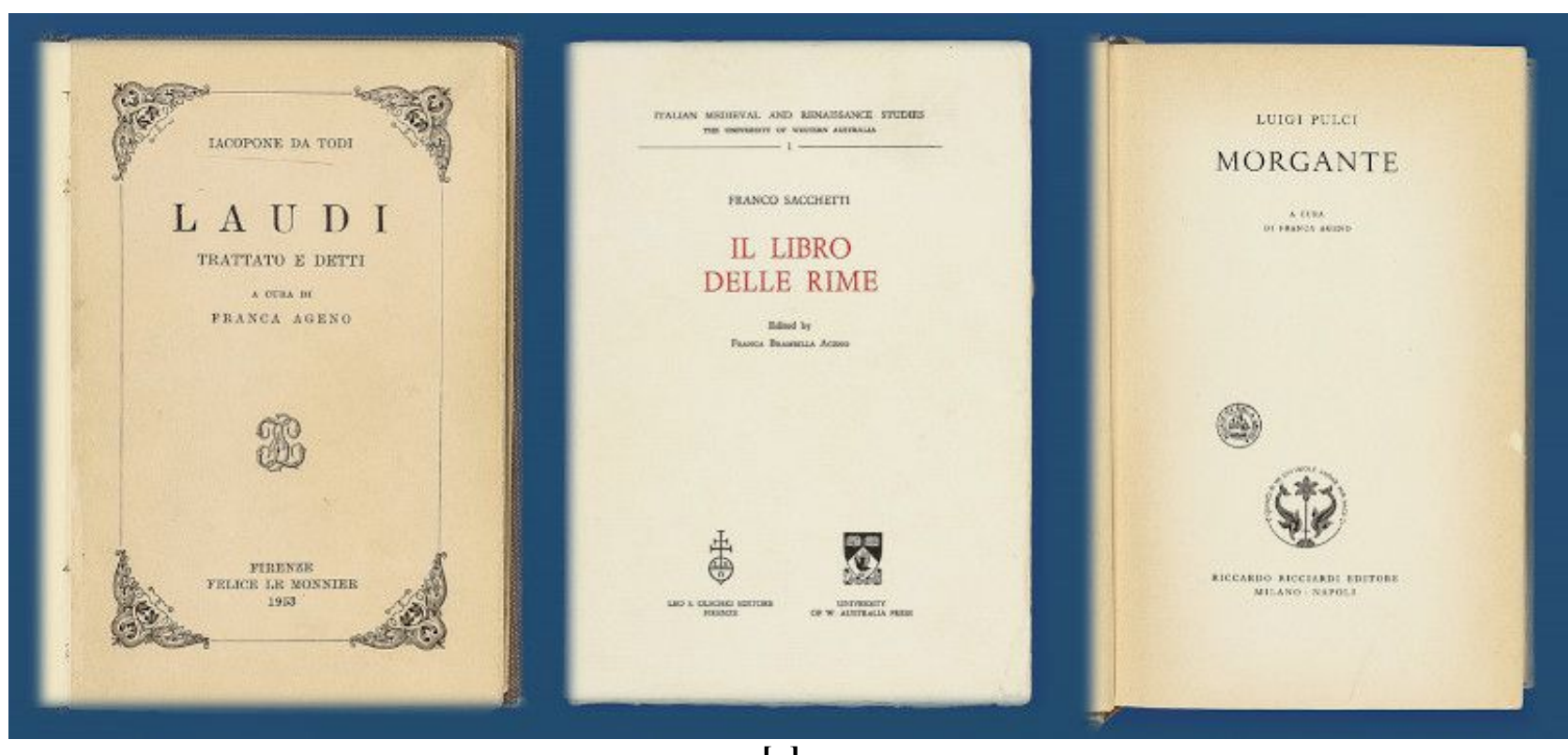

$[4]$

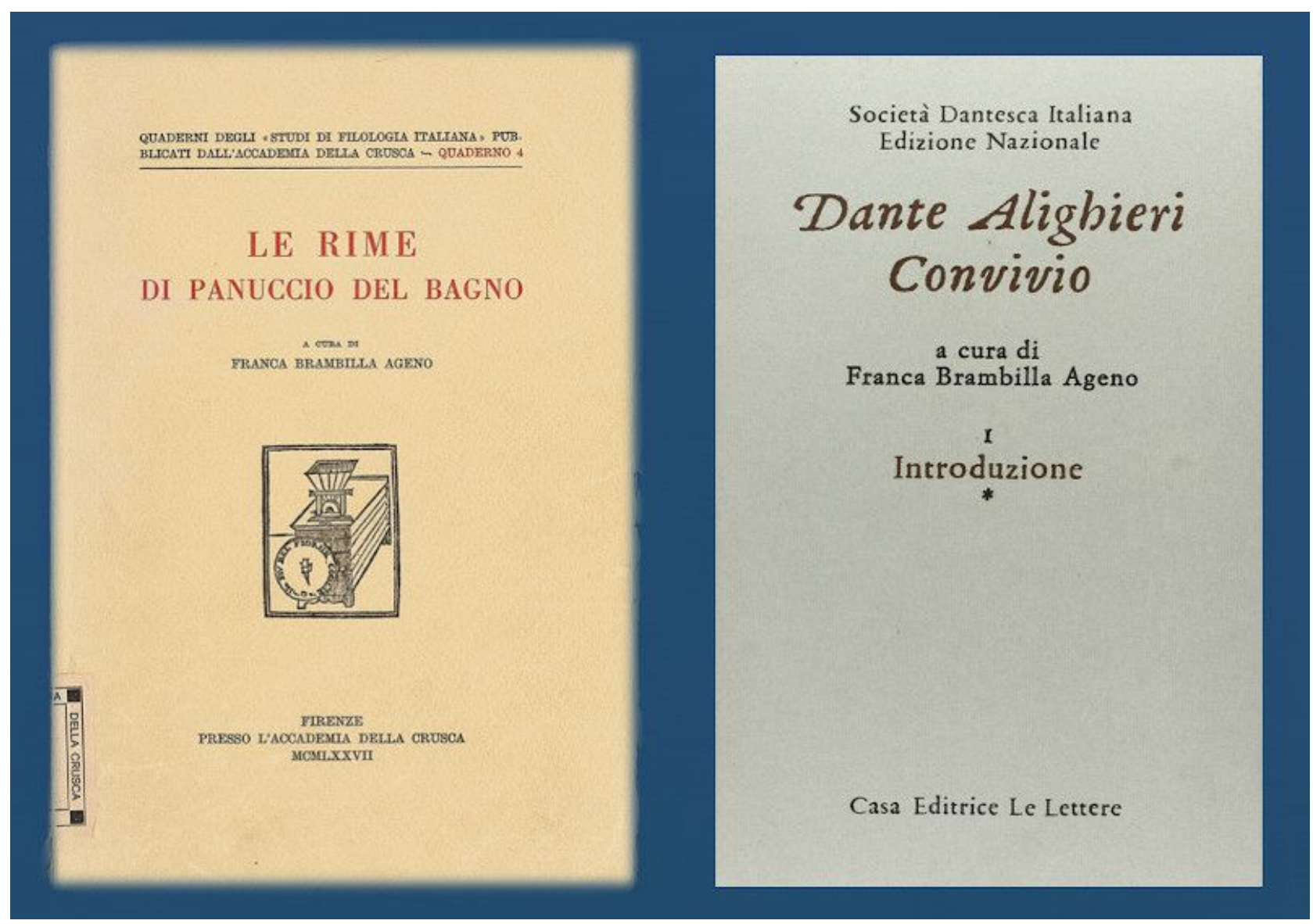



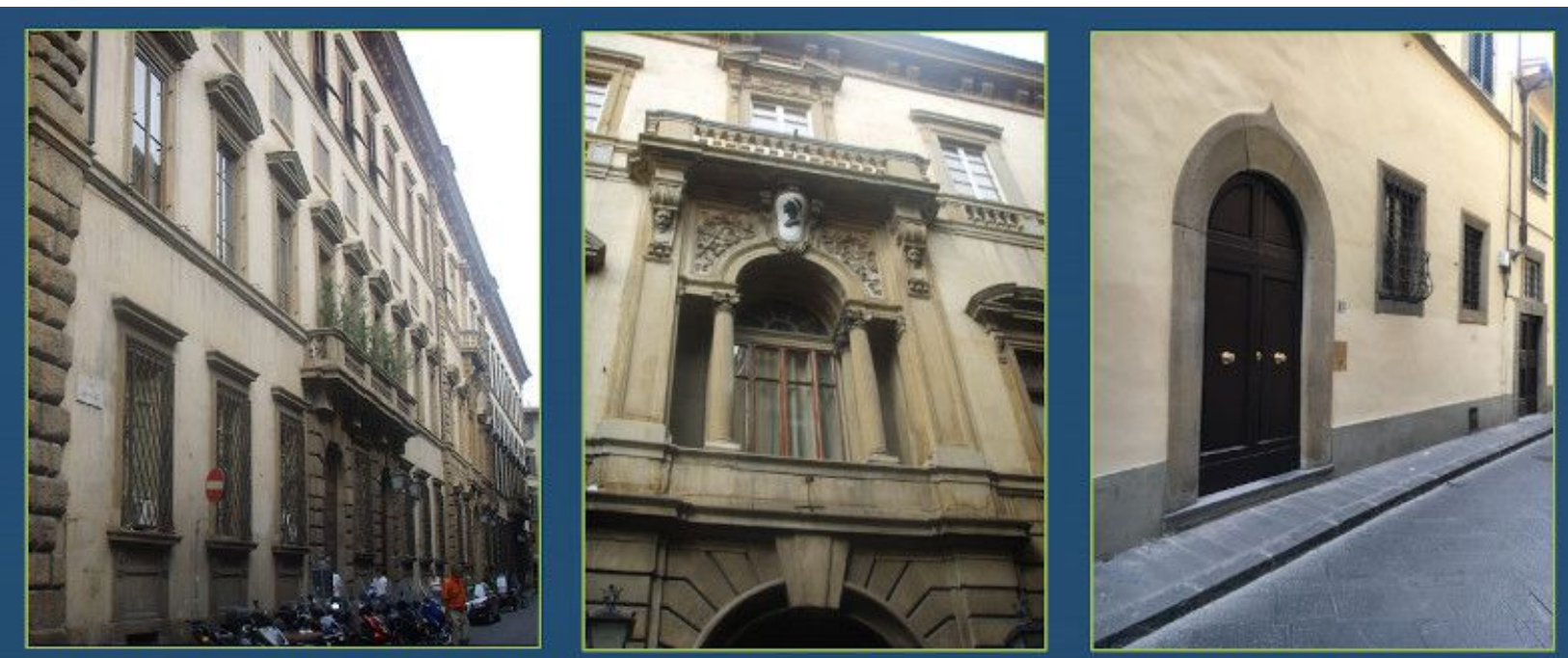

- Palamo Pucci, via de' Pucci, 4, Firenze (sede dell'Accademia della Crusca, febbraio 1938 - giugno 1940)

- Facciata del Palazzo Pucci (particolare)

- L'ingresso dell'abitazione di Franca Ageno, via Borgo Pinti 15, Firenze

[6]

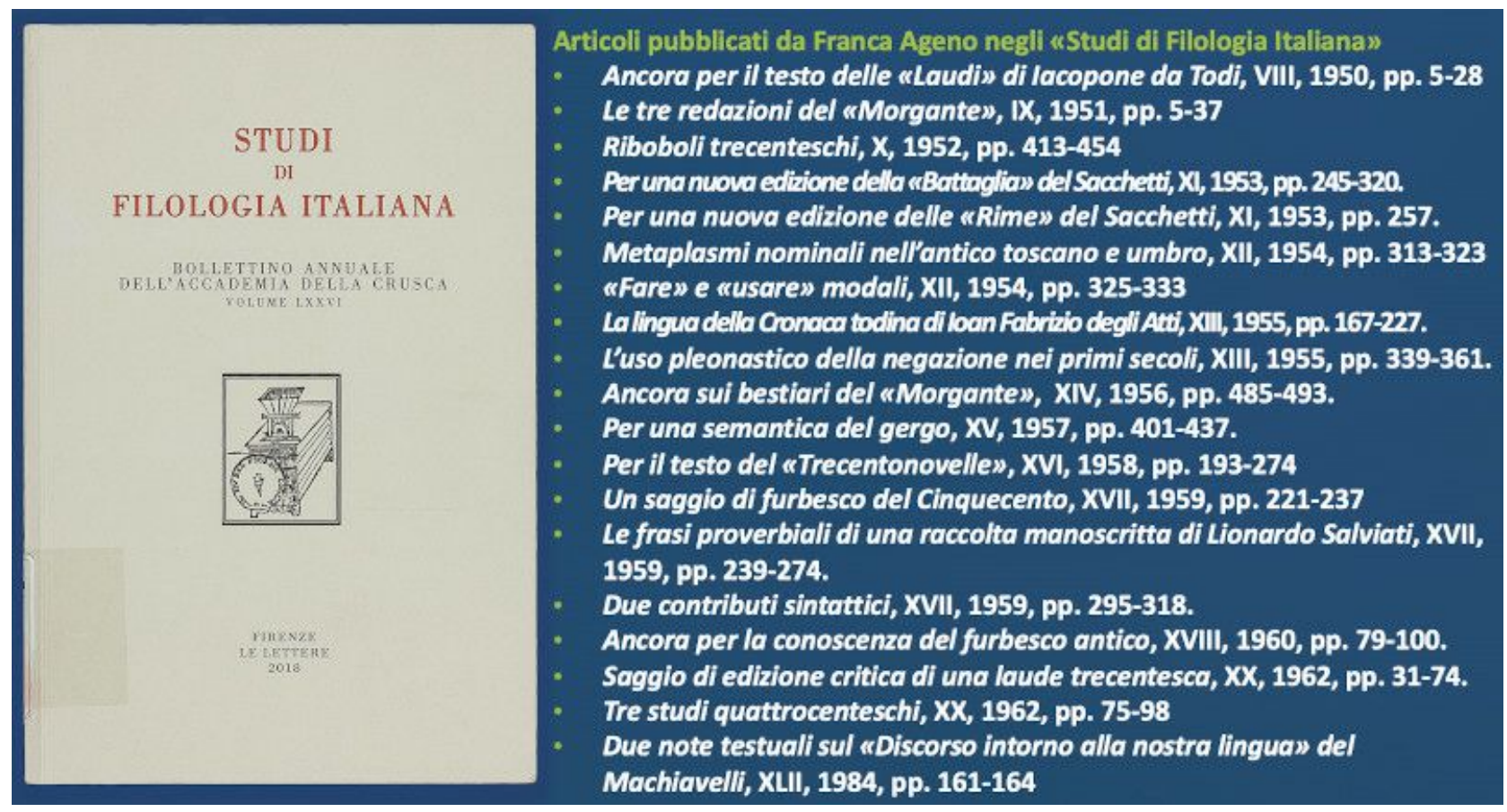

[7] 


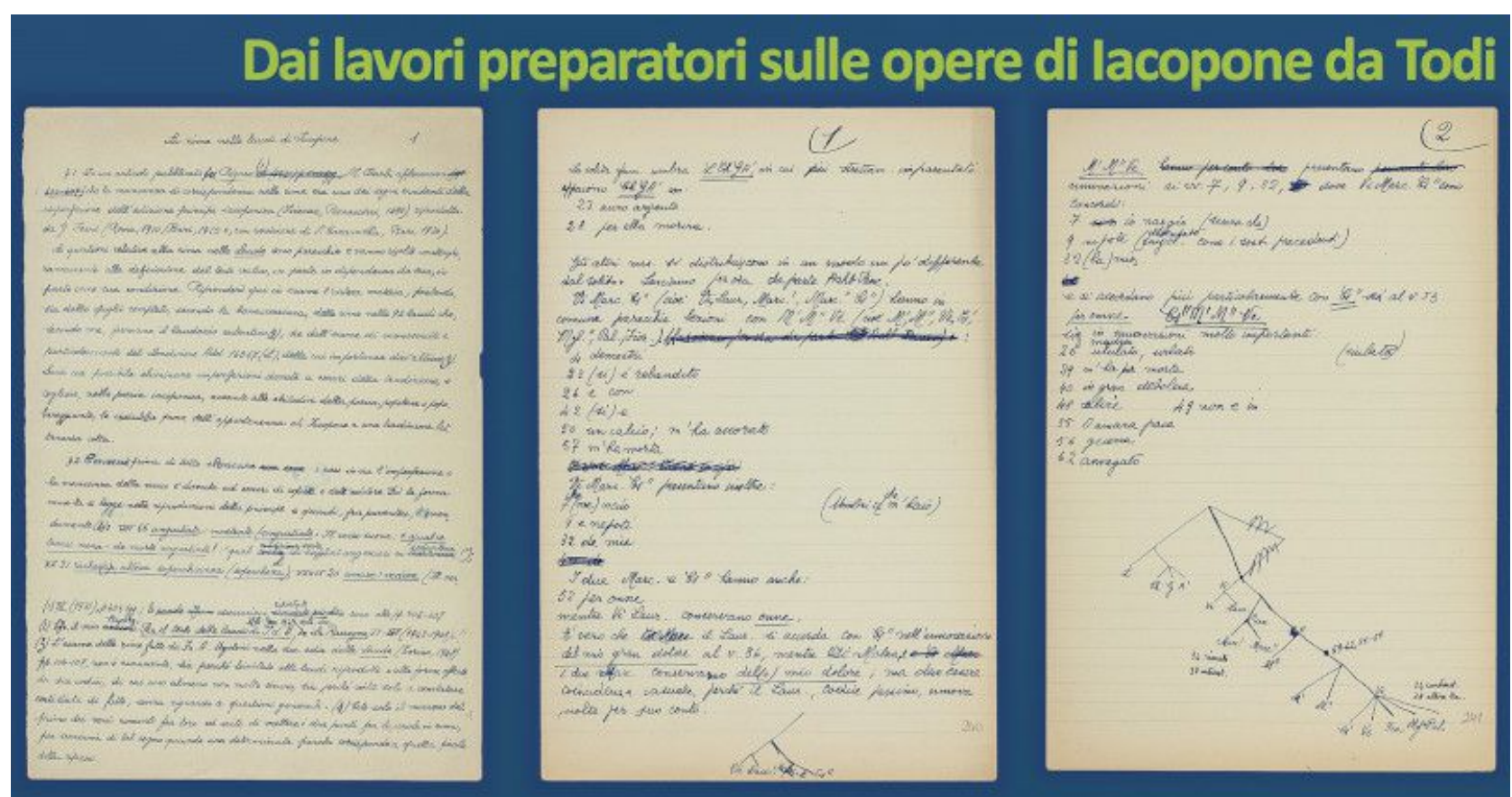

[8]

\section{Dalle carte sul Morgante di Pulci: appunti su Margutte}
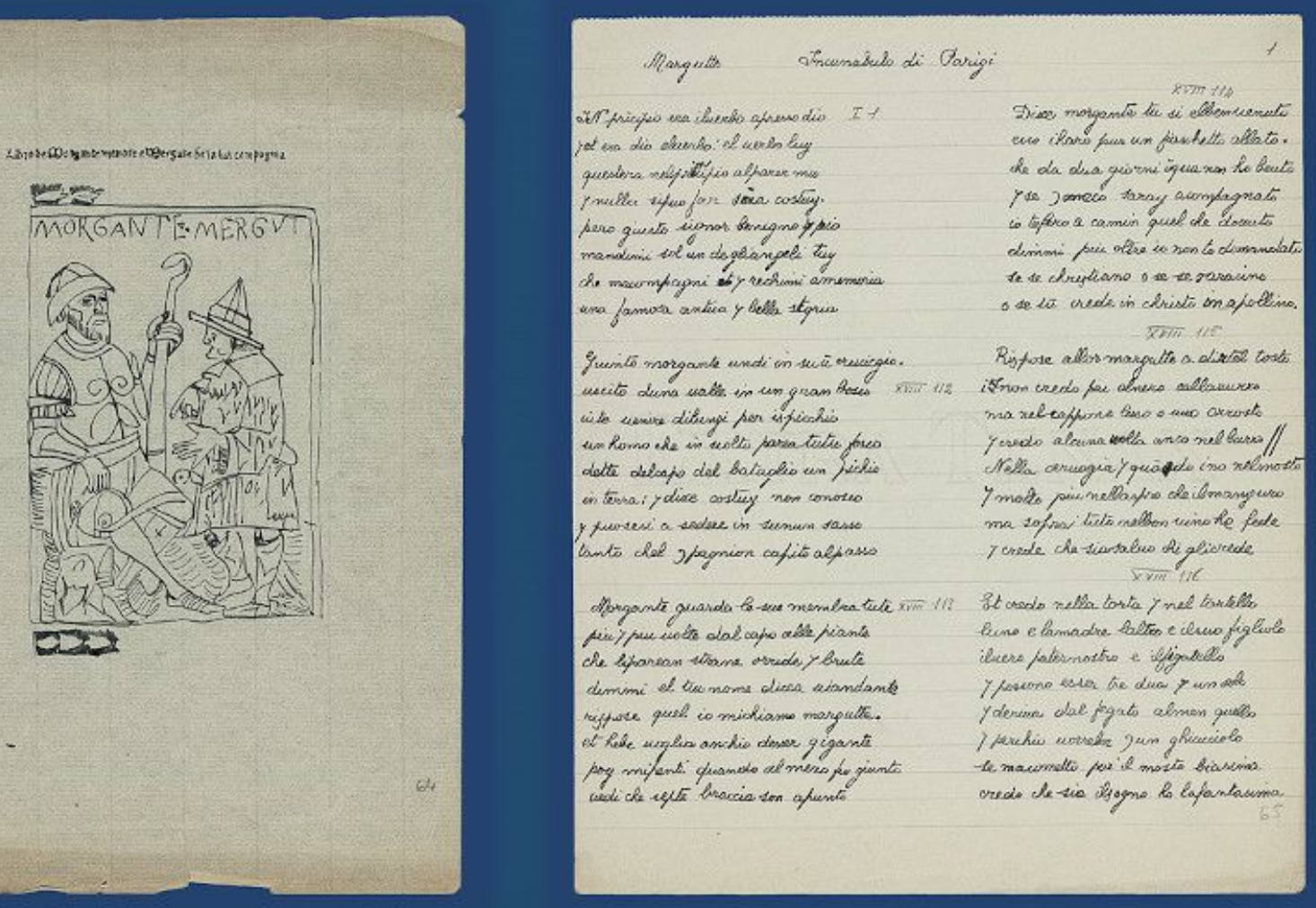

[9] 


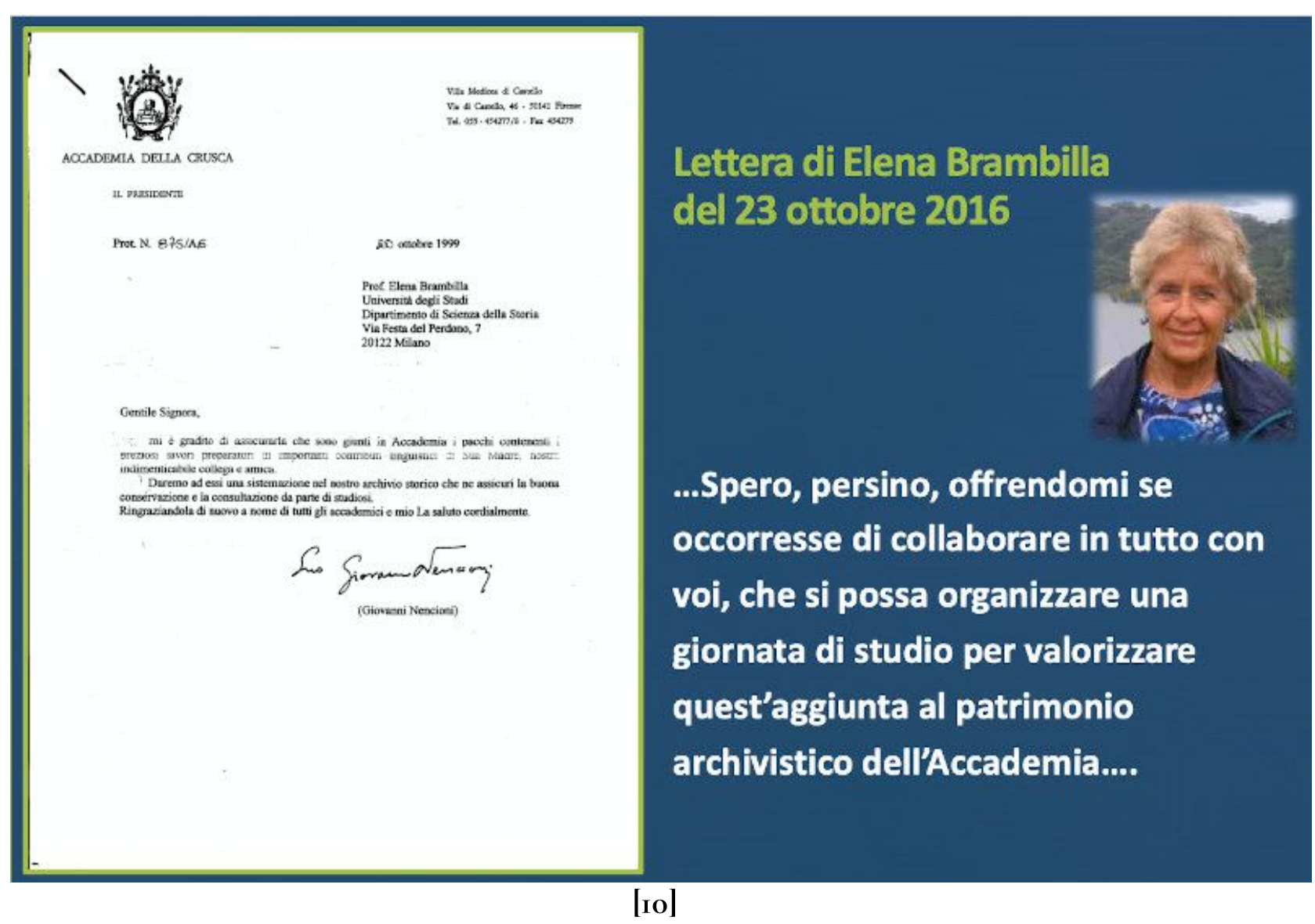

\section{Cita come:}

Elisabetta Benucci, Franca Brambilla Ageno e l'Accademia della Crusca, "Italiano digitale", 2019, XI, 2019/4 (ottobre-dicembre)

DOI: $10.35948 / 2532-9006 / 2020.3161$

Copyright 2019 Accademia della Crusca

Pubblicato con licenza creative commons CC BY-NC-ND 